T. Hida and L. Streit

Nagoya Math. J.

Vol. 68 (1977), 21-34

\title{
ON QUANTUM THEORY IN TERMS OF WHITE NOISE*
}

\author{
T. HIDA AND L. STREIT
}

\section{§1. The canonical representation}

It has often been pointed out that a much more manageable structure is obtained from quantum theory if the time parameter $t$ is chosen imaginary instead of real. Under a replacement of $t$ by $i \cdot t$ the Schrödinger equation turns into a generalized heat equation, time ordered correlation functions transform into the moments of a probability measure, etc. More recently this observation has become extremely important for the construction of quantum dynamical models, where criteria were developed by $\mathrm{E}$. Nelson, by K. Osterwalder and R. Schrader and others [8] which would permit the reverse transition to real time after one has constructed an imaginary time ("Euclidean") model.

The discussion of solutions for the heat equation

$$
\left(\partial_{t}-\partial_{x}^{2}+\lambda V(x)\right) \psi(x, t)=0
$$

may be reduced to that of certain integrals with respect to the Wiener measure $\mu_{B}$ for Brownian motion $B(t)$ of the general form

$$
E_{\lambda} F=\mathscr{N}_{\lambda} \int F[x] e^{-\lambda \int_{0}^{T} V(x(t)) d t} d \mu_{B}(x) .
$$

Alternatively one may consider white noise $\chi(t)$ as the basic stochastic process, realizing Brownian motion as

$$
B(t)=\int_{0}^{t} \chi(s) d s .
$$

In a recent paper [3] H. Ezawa, J. R. Klauder and C. A. Shepp [EKS] have proposed a new strategy for the calculation of expressions such as equation (2). The right side of (2) amounts to an average of the func-

Received September 29, 1976.

* This work was done at Zentrum für interdisziplinäre Forschung of Bielefeld University under the support of DFG. 
tional $F$ over Brownian motion sample paths with a weight factor to take into account the interaction $\lambda V$. EKS instead express $E_{\lambda} F$ as an unweighted average of $F$ over paths $R_{\lambda} x$ distorted in such a way that the mapping $R_{\lambda}$ incorporates the effect of the interaction

$$
E_{\lambda} F=\int F\left[R_{\lambda} x\right] d \mu_{B}(x)
$$

Among the results of EKS it is particularly worth emphasizing that the relation

$$
y=R_{\lambda} x
$$

remains well-defined-and that $y$-averaging still produces the correct result-when one passes to limits (such as $T \rightarrow \infty$ in equation (2)) where the original expression (2) fails to hold because the limit of

$$
\mathscr{N}_{2} e^{-\lambda \int_{0}^{T} V(x(t)) d t}
$$

ceases to be $\mu_{B}$-measurable so that it can no more serve as a RadonNikodym derivative to relate the $d \mu_{B}(x)$ to an "interacting" measure $d \nu(x)$. In the physicist's terminology the EKS formulation of dynamics

$$
y=R_{\lambda} x
$$

survives the removal of cutoffs while the Feynman-Kac formula (2) does not!

As EKS point out, this approach raises very interesting quations (and indeed even indicates the answer [1]) regarding the existence problem for certain types of stochastic differential equations. In this note we shall focus on Gaussian processes where that particular problem is well under control.

We wish to take into account the case of non-equivalent measures: the measure $\nu$ with respect to which we want to average the functional $F$ may well not be related to the white noise measure by a weight factor. Therefore we shall address ourselves to the discussion of maps $R$ such that

$$
\int F[x] d \nu(x)=\int F[R x] d \mu_{x}(x)
$$

where $\chi$ is one- or more-dimensional parameter white noise and $\nu$ is some 
Gaussian measure.

For a given $\nu, R$ is far from being unique. As an example consider the Ornstein-Uhlenbeck process given by the characteristic functional

$$
C_{\omega}(\xi)=\int e^{i\langle x, \xi\rangle} d \nu(x)=e^{-\frac{1}{2}\langle\xi, K \xi\rangle}
$$

with $(K \xi)(t)=\omega^{-1} \int e^{-\omega|t-s|} \xi(s) d s$. One possible realization of this process such that

$$
C_{\omega}(\xi)=\int e^{i\langle R x, \xi\rangle} d \mu_{\chi}(x)
$$

is obtained by setting

$$
R \chi(t)=R_{1} \chi(t)=C \int_{-\infty}^{\infty} K_{0}(\omega|t-s|) \chi(s) d s, \quad C=\frac{\sqrt{2}}{\pi},
$$

where $K_{0}(\cdot)$ is the Hankel function of imaginary argument.

To check this it suffices to verify that the covariance of $R_{x}$ equals that of the Ornstein-Uhlenback process since both are Gaussian:

$$
\begin{aligned}
\left\langle R_{\chi}\left(t_{1}\right), R_{\chi}\left(t_{2}\right)\right\rangle= & C^{2} \iint_{-\infty}^{\infty} K_{0}\left(\omega\left|t_{1}-s_{1}\right|\right) K_{0}\left(\omega\left|t_{2}-s_{2}\right|\right) \\
& \times\left\langle\chi\left(s_{1}\right), \chi\left(s_{2}\right)\right\rangle d s_{1} d s_{2} \\
= & C^{2} \int_{-\infty}^{\infty} K_{0}\left(\omega\left|t_{1}-s\right|\right) K_{0}\left(\omega\left|t_{2}-s\right|\right) d s \\
= & \omega^{-1} e^{-\omega\left|t_{1}-t_{2}\right|}=K\left(t_{1}, t_{2}\right) .
\end{aligned}
$$

On the basis of a stochastic differential equation for $R_{\chi}$, EKS arrive at quite a different representatation, namely

$$
R \chi(t)=2^{1 / 2} \int_{-\infty}^{t} e^{-\omega(t-s)} \chi(s) d s
$$

This representation is distinguished from general $R$ (and, as we shall see from all others, too) by the fact that it is causal and causally invertible. In terms of probability theory, such a property is said to be canonical.

The canonical property means the following: Let $\boldsymbol{B}_{t}(\chi)$ be the smallest sigma-field with respect to which all the $\langle x, \xi\rangle$ 's with $\operatorname{supp}(\xi) \subset[-\infty, t)$ are measurable. Suppose a Gaussian process $y$ is given by 


$$
y(t)=\int_{-\infty}^{t} F(t-s) \chi(s) d s .
$$

Then we can define $\boldsymbol{B}_{t}(y)$ in a similar manner to $\boldsymbol{B}_{t}(\chi)$. The representation (12) of $y$ in terms of $\chi$ is said to be canonical if

$$
\boldsymbol{B}_{t}(\chi)=\boldsymbol{B}_{t}(y) \quad \text { for every } t \text {. }
$$

Needless to say that there are many expressions of the form (12) such that the Gaussian process $y$ has the same covariance function $K\left(t_{1}, t_{2}\right)$ as the $R_{\chi}$. Among them there is only one representation satisfying (13), that is, the canonical representation is unique (see, e.g., T. Hida [5]).

The uniqueness as well as the existence of the canonical representation holds for more general stationary Gaussian processes that are purely non-deterministic (a process $y$ is called purely non-deterministic if and only if the sigma-field $\bigcap_{t} \boldsymbol{B}_{t}(y)$ is trivial).

A counterpart of the canonical representation is the backward canonical representation of a Gaussian process. Let $y(t)$ be given by

$$
y(t)=\int_{t}^{\infty} G(t-s) \chi(s) d s
$$

and let $B^{t}(\chi)$ be the smallest sigma-field with respect to which all the $\langle\chi, \xi\rangle$ 's with $\operatorname{supp}(\xi) \subset[t, \infty)$ are measurable. Similarly one defines the sigma-field $\boldsymbol{B}^{t}(y)$. The representation (14) of $y$ is called backward canonical if

$$
\boldsymbol{B}^{t}(y)=\boldsymbol{B}^{t}(\chi) \quad \text { for every } t
$$

Uniqueness and existence can be discussed in exactly the same manner as the canonical representation by interchanging future and past [5]. In particular the Fourier transforms of $F$ and $G$ are related via

$$
\overline{\hat{F}}(\lambda)=\hat{G}(\lambda) \text {. }
$$

\section{§2. Markov properties and $T$-positivity}

The Markov property of stochastic processes is of particular interest as an input to Nelson's reconstruction of relativistic fields from Euclidean ones, i.e. from certain stationary stochastic processes.

As is well known a process is said to be (simple) Markov if

$$
P(A \cap B / \boldsymbol{B}(x(t)))=P(A / \boldsymbol{B}(x(t))) P(B / \boldsymbol{B}(x(t)))
$$


for any $A \in \boldsymbol{B}_{t}$ and $B \in \boldsymbol{B}^{t}$. Here $\boldsymbol{B}(x(t))$ denotes the $\sigma$-field generated by $x(t)$ and we shall use $B=\bigvee_{t} B_{t}$.

It is known that a conditional expectation like $E\left(z / \boldsymbol{B}_{t}\right)$ of $z$ in $L^{2}(\Omega, \boldsymbol{B}, P)$ is the orthogonal projection of $z$ down to the subspace $L^{2}\left(\Omega, \boldsymbol{B}_{t}, P\right)$. However, one is concerned with only Gaussian random variables in this note, so that, if $z$ is a linear functional of the $x(t)^{\text {'s, the }}$ conditional expectation $E\left(z / \boldsymbol{B}_{t}\right)$ turns out to be the orthogonal projection to the closed linear subspace spanned by the $x(s), s \leq t$. The projection to this subspace is denoted by $E_{t}$. Similarly one denotes by $E^{t}$ the projection to the subspace spanned by the $x(s), s \geq t$. The symbol $E(t)$ is used to denote the projection to the closed linear subspace spanned by random variables measurable with respect to the sigma-field $\boldsymbol{B}(x(t))$.

For a stationary Gaussian process $E_{0}, E^{0}$ and $E(0)$ are usually denoted by $E_{-}, E_{+}$and $E_{0}$, respectively. We are now ready to describe the Markov property in terms of $E_{-}, E_{+}$and $E_{0}$. The following assertion immediately comes from (17). A stationary Gaussian process is (simple) Markov if and only if

$$
E_{-} x(t)=E_{0} x(t), \quad \text { for any } t \geq 0 .
$$

Or equivalently

$$
E_{-} E_{+}=E_{0} E_{+}
$$

in the space $L^{2}(\Omega, \boldsymbol{B}, P)$.

The stationary Gaussian Markov processes are exactly the Ornstein Uhlenbeck processes which solve

$$
L_{t} X(t)=\chi(t)
$$

where

$$
L_{t}=a_{0} \frac{d}{d t}+a_{1} \text { and } \quad a_{0} \cdot a_{1}>0
$$

A generalization is afforded by Gaussian processes obeying an $N$-th order differential operator of the form

$$
L_{t}=\sum_{k=0}^{N} a_{k} \frac{d^{N-k}}{d t^{N-k}}
$$

For an extension of this definition to the non-stationary case cf. [5]. In 
either case equation (17) generalizes to

$$
P(A \cap B / \boldsymbol{B}(t))=P(A / \boldsymbol{B}(t)) P(B / \boldsymbol{B}(t))
$$

where

$$
\boldsymbol{B}(t) \equiv \bigcap_{\varepsilon>0} \boldsymbol{B}(x(s): t-\varepsilon<s<t+\varepsilon)
$$

is the $\sigma$-field generated by the $x(s)$ in arbitrarily small neighbourhood of $t$. Furthermore one finds for the canonical kernel

$$
F(t, u)=\theta(t-u) \sum_{i=1}^{N} f_{i}(t) g_{i}(u) .
$$

Two distinct further generalizations are afforded by considering all those Gaussian processes which obey equation (25) - they are called N-ple Markov in the wide sense or those which obey equation (23)-they are called $\sigma$ Markov and are also characterized by equations such as (18) or (19) if we replace $\boldsymbol{B}(x(t))$ by $\boldsymbol{B}(t)$ in the definition of $E_{0}$.

Intuitively speaking the $\sigma$-Markov property of $x$ says that the future and the past become independent as soon as the present value as well as the values in an infinitesimal neighbourhood of the present are given.

Let $C_{x}(\xi), \xi \in \mathscr{S}$, be the characteristic functional of a mean continuous (in $t$ ) stationary Gaussian process $x=\{x(t) ; t \in R\}$ with $E(x(t)) \equiv 0$. Then $C_{x}(\xi)$ can be expressed in the form

$$
C_{x}(\xi)=\exp \left[-\frac{1}{2}\langle\xi, \gamma \xi\rangle\right],
$$

where $\gamma(t)$ is the covariance function of $x$ :

$$
\gamma(t)=E(x(t+s) x(s))
$$

As an example of the expression (26), one sees the formula (9).

Assume that $x$ is purely non-deterministic, that is, $\bigcap_{t} \boldsymbol{B}_{t}$ is trivial. Then the covariance function admits a spectral representation of the form

$$
r(t)=\int e^{i t \lambda} f(\lambda) d \lambda
$$

with the property that

$$
\left|\int \frac{\log f(\lambda)}{1+\lambda^{2}} d \lambda\right|<\infty
$$


One is given the following relationship

$$
f(\lambda)=|\hat{F}(\lambda)|^{2}=|\hat{G}(\lambda)|^{2}
$$

where $\hat{F}$ and $\hat{G}$ is the Fourier transform of $F$ in (12) and $G$ in (14), respectively. This proves that

1) $x$ is $N$-ple Markov in the restricted sense if and only if

$$
f(\lambda)=\frac{\text { const. }}{|P(i \lambda)|^{2}},
$$

where $P$ is a polynomial of degree $N$ without zeroes in the lower half $\lambda$-plane.

2) $x$ is $N$-ple Markov in the wide sense if and only if

$$
f(\lambda)=\left|\frac{Q(i \lambda)}{P(i \lambda)}\right|^{2}
$$

where $P$ and $Q$ are polynomials of degree $N$ and at most $N-1$, respectively, again without zeroes in the lower half $\lambda$-plane. (T. Hida [5]).

3) $x$ is $\sigma$-Markov if and only if $1 / f(\lambda)$ is an entire function of infraexponential type. (Y. Okabe [7]).

Yet another generalization of the simple Markov property was proposed by Hegerfeldt [4] since in the Euclidean field theory context it suffices to establish the existence of a corresponding Wightman theory. A time reflection operator $T$ may be defined in the Hilbert space $L^{2}(\Omega$, $\boldsymbol{B}, P$ ) of a Gaussian process $y$ by setting

$$
T 1=1 \in L^{2}(\Omega, B, P) \quad \text { and } \quad T y(t) T^{-1}=y(-t)
$$

Hegerfeldt's T-positivity condition is

$$
E_{+} T E_{+} \geq 0 \text {. }
$$

By standard arguments it is sufficient (and of course necessary) to establish that this holds on the closed subspace $L(y)$ spanned by the $y(t)$, $t \in \boldsymbol{R}$.

A dense linear subspace of $E_{+} L(y)$ is provided by vectors

$$
\sum a_{\nu} y\left(t_{\nu}\right) \text { with } t_{\nu} \geq 0, \quad a_{\nu} \in C
$$

so that $T$-positivity for a Gaussian process becomes equivalent to 


$$
\sum \bar{a}_{\nu} a_{\mu} E\left(y\left(t_{\nu}\right) y\left(-t_{\mu}\right)\right) \geq 0
$$

or in terms of the covariance matrix $\gamma$

$$
\sum \bar{a}_{\nu} a_{\mu} \gamma\left(t_{\nu}+t_{\mu}\right) \geq 0 .
$$

It is worth pointing out that such stationary Gaussian processes can be completely classified: their covariance matrices form a convex cone spanned by those of the Ornstein-Uhlenbeck processes.

LEMMA. Let $\gamma(t), t \geq 0$ be a bounded complex function such that $\forall n \geq 0$ and $a_{v} \in \boldsymbol{R} \sum^{n} a_{\mu} a_{\nu} \gamma\left(t_{\mu}+t_{\nu}\right) \geq 0$ ("T-positivity"). Then $\gamma(t)$ is completely monotonic, i.e. $\gamma(t)=\int_{0}^{\infty} e^{-\lambda t} d \alpha(\lambda)$ where $\alpha$ is a finite Borel measure.

Proof. By Bernstein's theorem [9] the existence of the above integral representation is equivalent to the inequality

$$
\sum_{m=0}^{N}(-1)^{m}\left(\begin{array}{l}
N \\
m
\end{array}\right) r(t+m h) \geq 0 \quad \forall t, h \geq 0 .
$$

It is useful to introduce the difference operator $\Delta_{n}$ such that

$$
\left(\Delta_{h} f\right)(t)=f(t+h)-f(t)
$$

in terms of which we can rewrite the above inequality in the form

$$
(-)^{N} \Delta_{h}^{N} \gamma(t) \geq 0 \text {. }
$$

The T-positivity, with the particular choice $a_{\nu}=(-)^{\nu}\left(\begin{array}{l}n \\ \nu\end{array}\right)$ and $t_{\nu}=\frac{1}{2} t$ $+\nu h$ yields

$$
0 \leq \sum_{\mu, \nu}(-)^{\nu+\mu}\left(\begin{array}{l}
n \\
\nu
\end{array}\right)\left(\begin{array}{l}
n \\
\mu
\end{array}\right) \gamma(t+(\nu+\mu) h)=\Delta_{h}^{2 n} \gamma(t) .
$$

It remains to show that $\forall n, \Delta_{h}^{2 n+1} \gamma$ is negative. To this end we consider the function

$$
g(t)=\Delta_{h}^{2 n} \gamma(t) \geq 0
$$

We know that $g(t)$ is bounded (as a finite sum of bounded functions) as well as convex:

$$
\Delta_{h}^{2} g(t)=\Delta_{h}^{2(n+1)} \gamma(t) \geq 0
$$


Hence $g(t)$ must be monotone decreasing, i.e.

$$
\Delta_{h} g(t) \leq 0 \text {. }
$$

Note in particular that all $T$-positive bounded functions are infinitely differentiable, and more importantly, they all are covariance matrices. From this we conclude immediately the

THEOREM. The covariance functions of stationary T-positive second order processes $y$ are of the form

$$
E(y(t+s) y(s))=\int_{0}^{\infty} e^{-|t| \lambda} d \alpha(\lambda)
$$

for some finite positive Borel measure $\alpha$, and any such measure gives rise to a T-positive Gaussian process.

Wide sense $N$-ple Markov processes arise from the measures $\alpha$ with $N$ point support

$$
\alpha\left(\lambda_{\nu}\right)=\alpha_{\nu}
$$

since they give rise to spectral densities

$$
f(\lambda)=\frac{1}{\pi} \sum_{\nu=1}^{N} \frac{\alpha_{\nu} \lambda_{\nu}}{\lambda^{2}+\lambda_{\nu}^{2}} .
$$

Conversely $N$-ple Markov processes are $T$-positive if only

$$
f(\lambda)=\sum_{\nu=1}^{N} \frac{\beta_{\nu}}{\lambda^{2}+\lambda_{\nu}^{2}} \quad \beta_{\nu}>0
$$

which (for $N>1$ ) is never the case for restricted sense $N$-ple Markov processes.

It is interesting to exhibit the way in which the reflection operator $T$ acts on the innovation $\chi_{-}$in the canonical representation

$$
y(t) \cdot=\int_{-\infty}^{t} F(t-u) \chi_{-}(u) d u
$$

as well as on its counterpart $\chi_{+}$in

$$
y(t)=\int_{t}^{\infty} G(t-u) \chi_{+}(u) d u .
$$

Here we assume that $y$ is mean continuous and purely non-deterministic. $y$ has the spectral representation 


$$
y(t)=\int e^{i t \lambda} Z(\lambda) d \lambda
$$

so that

$$
T Z(\lambda)=\bar{Z}(\lambda)
$$

By taking Fourier transforms of the canonical representations one finds

$$
\hat{\chi}_{-}(\lambda)=\bar{Z}(\lambda) / \hat{F}(\lambda) \quad \hat{\chi}_{+}(\lambda)=\bar{Z}(\lambda) / \hat{G}(\lambda) .
$$

Using $\overline{\hat{F}}(\lambda)=\hat{G}(\lambda)$ it follows that

$$
T \hat{\chi}_{+}(\lambda)=\overline{\hat{\chi}_{-}(\lambda)},
$$

so that finally the two innovations $\chi_{ \pm}$are related through

$$
T \chi_{+}(u)=\chi_{-}(-u) \text {. }
$$

\section{§3. Euclidean fields in terms of white noise}

Relativistic free scalar fields associated with the Hamiltonian

$$
H_{0}=\frac{1}{2} \int d^{s} x: \pi^{2}(x)+(\nabla \phi)^{2}(x)+m^{2} \phi^{2}(x):
$$

give rise to Euclidean fields $\Phi$ with the characteristic functional

$$
C_{0}(\xi)=\left\langle\Omega, e^{i \Phi(\xi)} \Omega\right\rangle=e^{-\frac{1}{2\left(\xi,\left(-\Delta_{t}, x+m^{2}\right)-1 \xi\right)}} \quad \xi \in \mathscr{S}\left(R^{s+1}\right) .
$$

Associated with this characteristic functional is a probability measure $\nu_{0}$ on the space $\mathscr{S}^{\prime}=\mathscr{S}^{\prime}\left(R^{s+1}\right)$ such that

$$
C_{0}(\xi)=\int_{y_{0} \in \mathscr{S}^{\prime}} e^{i\left\langle y_{0}, \xi\right\rangle} d \nu_{0}\left(y_{0}\right)
$$

Thus one is given a probability measure space $\left(\mathscr{S}^{\prime}, \underline{\underline{B}}, \nu_{0}\right)$, where $\underline{\underline{B}}$ is the sigma-field generated by the cylinder sets. Each member $y_{0}$ in $\mathscr{S}^{\prime}$ is now viewed as a sample path of a random field having the characteristic functional $C_{0}(\xi)$.

On the other hand, there is a measure space $\left(\mathscr{S}^{\prime}, \underline{\underline{B}}, \mu_{x}\right)$, call it white noise, given by the characteristic functional

$$
C_{\chi}(\xi)=e^{-\|\xi\|^{2} / 2} .
$$

As before a sample path of white noise is denoted by $\chi$. Now arises an 
interesting problem asking how to describe $y_{0}$ in terms $\chi$ like the expression (5). The answer to this question is stated by the following theorem.

THEOREM. A sample path $y$ of the random field given by the characteristic functional $C_{0}$ is expressed in the form

$$
\frac{\partial}{\partial t} y_{0}(t, x)=-\omega_{0} y(t, x)+\chi(t, x)
$$

on the measure space $\left(\mathscr{S}^{\prime}, \underline{\underline{B}}, \mu_{\mathrm{x}}\right)$, where

$$
\omega_{0}=\sqrt{-\Delta_{x}+m^{2}} .
$$

Remark. One should note that the equation (58) is a stochastic differential equation in terms of generalized functions. Such an equation has been discussed by A. V. Balakrishnan [2] with a different flavour.

Proof of the theorem. For simplicity $s$ is assumed to be 1 throughout the proof.

a) Since $-\Delta_{x}+m^{2}>0$, it is possible to have its square root denoted by $\omega_{0}$. The domain of $\omega_{0}$ is rich enough, that is, wider than the Schwartz space $\mathscr{S}(R)$. Hence a semi-group $\left\{T_{t} ; t \geq 0\right\}$ is given by

$$
T_{t}=e^{-\omega_{0} t}, \quad t \geq 0
$$

and $T_{t}$ is continuous in $t$. Thus the integral

$$
y(t, x)=\int_{-\infty}^{t} T_{t-u} \chi(u, x) d u
$$

is well-defined. More precisely, taking $\xi$ in $\mathscr{S}(\boldsymbol{R})$,

$$
\int_{-\infty}^{t} d u \int_{R} d x\left(T_{t-u} \xi\right)(x) \chi(u, x)
$$

is defined and the integral is to be denoted by $y(t, \xi)$.

b) It is straightforward to verify that $y(t, x)$ given by (61) satisfies the stochastic differential equation. Namely $y$ is a version of $y_{0}$.

c) It remains to show that the characteristic functional of the random field $y$ is exactly equal to $C_{0}$. Since $y$ is Gaussian and has zero expectation, it suffices to compute the covariance function of $y(t, \xi)$. For $h>0$, one obtains 


$$
\begin{aligned}
& E\{y(t+h, \xi) y(t, \xi)\} \\
& \quad=E\left[\int_{-\infty}^{t+h} d u \int_{R^{s}} d x\left(T_{t+h-u} \xi\right)(x) \chi(u, x) \cdot \int_{-\infty}^{t} d u^{\prime} \int_{R^{s}} d x^{\prime}\left(T_{t-u^{\prime}} \xi\right)\left(x^{\prime}\right) \chi\left(u^{\prime}, x^{\prime}\right)\right] \\
& \quad=\int_{-\infty}^{t} d u \int d x\left(T_{t+h-u} \xi\right)(x)\left(T_{t-u} \xi\right)(x) .
\end{aligned}
$$

Now change the order of integration and use the Fourier tranform in $x$ to rewrite the above integral in the form

$$
\begin{aligned}
& \int_{0}^{\infty} d u \int e^{-\sqrt{p^{2}+m^{2}}(h+u)} \hat{\xi}(p) \cdot e^{-\sqrt{p^{2}+m^{2}} u} \overline{\hat{\xi}(p)} d p \\
& =\int \frac{1}{2 \sqrt{m^{2}+p^{2}}} e^{-\sqrt{p^{2}+m^{2} h}}|\hat{\xi}(p)|^{2} d p .
\end{aligned}
$$

Similar computations lead us to prove

$$
E\{y(t+h, \xi) y(t, \xi)\}=\frac{1}{2 \sqrt{2 \pi}} \iint e^{-i p_{0} h} \frac{|\hat{\xi}(p)|^{2}}{p_{0}^{2}+p^{2}+m^{2}} d p d p_{0}
$$

for general $h(\geq 0$ or $\leq 0)$.

Further, setting $y(\xi, \eta)=\int \eta(t) y(t, \xi) d t$ one obtains

$$
E\left(y(\xi, \eta)^{2}\right)=\frac{1}{4 \pi} \iint \frac{\left|\hat{\eta}\left(p_{0}\right) \hat{\xi}(p)\right|^{2}}{p_{0}^{2}+p^{2}+m^{2}} d p_{0} d p,
$$

which extends to

$$
E\left(y(f)^{2}\right)=\frac{1}{4 \pi} \iint \frac{\left|\hat{f}\left(p_{0}, p\right)\right|^{2}}{p_{0}^{2}+p^{2}+m^{2}} d p_{0} d p, \quad f \in \mathscr{S}\left(R^{2}\right)
$$

This shows that the characteristic functional of $y$ is $C_{0}$.

q.e.d.

Observe now the integrand of the expression (63). The square root of the density function $\left(p_{0}^{2}+p^{2}+m^{2}\right)^{-1}$ may be taken to be $\left(-i p_{0}^{2}+\sqrt{p^{2}+m^{2}}\right)^{-1}$, which corresponds to the differential operator $\frac{\partial}{\partial t}+\omega_{0}$ in the $(t, x)$-space. $\left(-i p_{0}+\sqrt{p^{2}+m^{2}}\right)^{-1}$ gives the canonical representation in the sense that

$$
\boldsymbol{B}_{t}(y)=\boldsymbol{B}_{t}(\chi) \quad \text { for every } t
$$

where $\boldsymbol{B}_{t}(y)$ (or $\boldsymbol{B}_{t}(\chi)$ ) is the sigma-field generated by $y(s, \xi)$ (or $\chi(s, \xi)$ ), $s \leq t, \xi \in \mathscr{S}(\boldsymbol{R})$. Namely, the expression (61) is the canonical representation of $y$ with respect to $\chi$.

This formulation of dynamics in terms of a stochastic differential 
equation or a canonical representation like (58) or (61) is particularly remarkable for its stability under singular perturbations such as they arise naturally in relativistic quantum field theory. The introduction of such perturbations into a Hamiltonian (or a Feynman-Kac formula) requires more or less drastic regularizations or "cutoffs". Technically this restriction can be viewed as the requirement that the perturbed probability measure be absolutely continuous with respect to the original one. This condition is absent from the formulation in terms of transformed white noise.

To illustrate this point we shall discuss the formal interaction term.

$$
H_{I}=\frac{1}{2} \int d^{s} x g(x): \phi^{2}:(x)
$$

which gives rise to a characteristic functional for the Euclidean field of the form

$$
C_{g}(\xi)=e^{-\frac{1}{2}\left(\xi,\left(-\Delta_{t, x}+m^{2}+g(x)\right)-1 \xi\right)}, \quad \xi \in \mathscr{S}\left(R^{s+1}\right) .
$$

It is reasonable to assume that $g(x)$ is smooth, bounded and nonnegative. With this assumption one can proceed with the same argument as in the case of $C_{0}$. A sample path of the random field with the characteristic functional $C_{g}$ will be denoted by $y_{g}$. Set

$$
\omega_{g}=\sqrt{-\Delta_{x}+m^{2}+g(x)} .
$$

Then, it holds that

$$
\frac{\partial}{\partial t} y_{g}(t, x)=-\omega_{g} y_{g}(t, x)+\chi(t, x)
$$

The canonical representation with respect to $\chi$ is of the form

$$
y_{g}(t, x)=\int_{-\infty}^{t} T_{t-u}^{g} \chi(u, x) d u
$$

where $\left\{T_{t}^{g} ; t \geq 0\right\}$ is a semi-group given by

$$
T_{t}^{g}=e^{-\omega_{g} t}, \quad t \geq 0 .
$$

Concerning the relationship between $y_{0}$ and $y_{g}$, the following expression can be given by using (67): 


$$
y_{g}(t, x)=y_{0}(t, x)-\int_{-\infty}^{t} T_{t-h}^{g}\left(\omega_{g}-\omega_{0}\right) y_{0}(u, x) d u
$$

This implies

$$
\begin{aligned}
& \frac{\partial}{\partial t}\left\{y_{g}(t, x)-y_{0}(t, x)\right\} \\
& \quad=-\left(\omega_{g}-\omega_{0}\right) y_{0}(t, x)+\int_{-\infty}^{t} T_{t-u}^{g} \omega_{g}\left(\omega_{g}-\omega_{0}\right) y_{0}(u, x) d u .
\end{aligned}
$$

Finally it might be interesting to point out that, after replacing $g(x)$ with $\lambda g(x)$, the asymptotic behaviour of $y_{\lambda g}$ as $\lambda \downarrow 0$ can easily be discussed through the expression (68) or (69).

ACKNOWLEDGEMENTs. The authors are grateful to S. Albeverio for helpful discussions and to $\mathrm{H}$. Oodaira for pointing out the relevance of Bernstein's theorems.

\section{REFERENCES}

[1] S. Albeverio and R. Høegh-Krohn, Drichlet Forms and Diffusion Processes on Rigged Hilbert Spaces, Oslo Univ. Preprint, December 1975.

[2] A. V. Balakrishnan, Stochastic Optimization Theory in Hilbert Spaces-1. Applied Math. and Optimization 1 (1974), 97-120.

[ 3 ] H. Ezawa, J. R. Klauder and L. A. Shepp, A Path Space Picture for FeynmanKac Averages. Ann. of Phys. 88 (1974), 588-620.

[4] G. C. Hegerfeldt, From Euclidean to Relativistic Fields and on the Notion of Markoff Fields. Commun. Math. Phys. 35 (1974), 155-171.

[5] T. Hida, Canonical Representations of Gaussian Processes and their Applications, Mem. Coll. Sci. Univ. Kyoto, Ser. A33 (1960), 109-155.

T. Hida, "Functionals of Brownian Motion II", ZiF Lectures 1976.

[ 6 ] — Stationary Stochastic Processes. Princeton Univ. Press 1970.

[ 7$]$ Y. Okabe, Stationary Gaussian Processes with Markovian Property and M. Sato's Hyperfunctions. Japanese Journ. of Math. 41 (1973), 69-112.

[ 8 ] for a review of B. Simon: The $P(\phi)_{2}$ Euclidean (Quantum) Field Theory, Princeton Univ. Press 1974.

[9] e.g. D. V. Widder: The Laplace Transform. Princeton Univ. Press 1946.

Department of Mathematics

Nagoya University,

Fakultät für Physik

Universität Bielefeld 